\title{
B Vitamin Intake and the Risk of Colorectal Cancer Development: A Systematic Review and Meta-Analysis of Observational Studies
}

\author{
Eva Kiss ${ }^{1}$, Dorottya Muhl ${ }^{1}$, Reka Mohacsi ${ }^{1}$, Magdolna Dank ${ }^{1}$, Istvan Takacs ${ }^{2}$ and Zsuzsanna \\ Nemeth $^{2 *}$

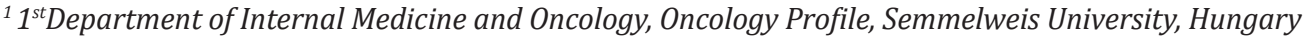 \\ ${ }^{2} 1^{\text {st }}$ Department of Internal Medicine and Oncology, Semmelweis University, Hungary
}

*Corresponding author: Zsuzsanna Nemeth, $1^{\text {st }}$ Department of Internal Medicine and Oncology, Semmelweis University, Hungary-1083 Budapest, Korányi Sándor u. 2/a, Hungary

ARTICLE INFO

Received: 幽 December 12, 2021

Published: 蔧January 07, 2022

Citation: Eva Kiss, Dorottya Muhl, Reka Mohacsi, Magdolna Dank, Istvan Takacs and Zsuzsanna Nemeth. B Vitamin Intake and the Risk of Colorectal Cancer Development: A Systematic Review and Meta-Analysis of Observational Studies. Biomed J Sci \& Tech Res 40(5)-2022. BJSTR. MS.ID.006516.

Abbreviations: 5,10-THF: 5-Methyltetrahydrofolate; CES: Combined Effect Size; CI: Confident Interval; CRC: Colorectal Cancer; FFQ: Food Frequency Questionnaire; HR: Hazard Ratio; MTHFR: Methylenetetrahydrofolate Reductase; NOS: Newcastle-Ottawa Scale; OR: Odds Ratio; PI: Prediction Interval; PRISMA: Preferred Reporting Items for Systematic Reviews and Meta-Analyses; QoL: Quality of Life; RR: Relative Risk; SAH: S-Adenosylhomocysteine; SAM: S-Adenosylmethionine; SNP: Single Nucleotide Polymor-

\section{ABSTRACT}

Background: Cancer research has increased interest in lifestyle factors. These can affect colorectal cancer burden, which is the second common cause of cancer death worldwide, although $40 \%$ of the cases would be preventable.

Aim: We aimed to conduct a systematic review and meta-analysis on relationship between B vitamin intake and colorectal cancer where 14 publications were analysed.

Methods: We carried out a systematic search of scientific literature based on PRISMA statements. Combined effect size (CES), confident interval (CI), prediction interval (PI), I2 and publication bias were calculated during statistical analysis of selected studies.

Results: Combined effect sizes showed inverse association between higher intake of vitamin B2 (CES = 0.90; CI95\% $0.83-0.97$; $2=0.00 \%, p=0.910$ ), B6 (CES = 0.80; CI95\% 0.68-0.92; I2 $=9.17 \%, p=0.359$ ) and CRC. We could not confirm the higher dietary intake of vitamin B12 reducing the risk of CRC. Vitamin B2 and B6 could compensate the effect of MTHFR C677T polymorphism (CES $=0.81$; CI95\% $0.64-0.98$; $\mathrm{I} 2=0.00 \%, \mathrm{p}=0.515$ ) as well.

Conclusion: Our results suggest that optimal intake of vitamin B2 and B6 could be important dietary factors in prevention of CRC. The association with vitamin B12 is inconsistent as its bioavailability is affected by other lifestyle factors. Vitamin B2 and B6 could influence MTHFR enzyme activity, therefore these vitamins might be incorporated into screening process of CRC with recommendations for specific diet.

Keywords: B Vitamins; Dietary Intake; Colorectal Cancer; Prevention; MTHFR Gene

\section{Introduction}

Recent cancer research has increased interest in lifestyle factors like diet, physical activity, stress level or habits which are influenced by socio-economical state and socio-behavioral factors as well. They affect the human physiology and have significant impact on the development of cancer and other diseases [1-3]. According to GLOBOCAN 2020, colorectal cancer (CRC) is the third 
most frequent cancer type and the second most common cause of cancer death worldwide, although around $40 \%$ of the cases would be preventable [4]. Countries with better and careful cancer prevention programs have more chance to fight against CRC [5]. Dietary intake of methyl donors (such as folate, choline, betaine, methionine and vitamin B2, B6 and B12) could have important role in cancer prevention by reducing the risk of cancer and could contribute to the success of cancer therapies and to reach better quality of life (QoL) of the patients [6-8]. Dietary methyl donors are food components, which provide methyl groups for the one-carbon metabolism, which consists of two main metabolic cycles: the folate cycle and the methionine cycle [9]. Methionine has a universal methyl group and can be added to several molecules; thus, its sufficient amount supports the normal DNA methylation [10]. It is also well known that inadequate DNA methylation may lead to development of cancer [6].

The optimal function of one-carbon metabolism requires specific vitamins as well as minerals. B vitamins are catalytic co-enzymes in these processes; therefore, they can influence the availability of methyl groups [10]. Moreover, B vitamins are important in energy-yielding metabolism, oxygen transport and neuronal functions. They play essential roles in basic metabolic pathways and fundamental cellular functions consequently have an impact on cognitive and psychological processes, including mental and physical fatigue $[7,11]$. Besides nutritional and other lifestyle factors, genetically determined components influence the development of CRC as well. One of these is the single nucleotide polymorphism (SNP) of the methylenetetrahydrofolate reductase (MTHFR) gene. MTHFR is involved in the one-carbon metabolism, where this enzyme activates folic acid. It has a common SNP at the position of 677 (MTHFR C677T). The heterozygous mutation (CT) results in a reduced enzyme activity around $65 \%$ of the normal level, while the homozygous (TT) mutation causes only $30 \%$ enzyme activity, and both reduce the level of DNA methylation [12-14].In this meta-analysis our aim was to systematically collect publicly available data, and summarize and update the scientific knowledge about the associations between dietary B2, B6 and B12 vitamin intake and the risk of CRC in adult patients, which has already published until 15th March 2021. Moreover, we aimed to highlight the importance of the need for standardization of the way how to explain the result of a meta-analysis as well.

\section{Materials and Methods}

\section{Study Characteristics}

Our systematic review and meta-analysis based on Preferred Reporting Items for Systematic Reviews and Meta-Analyses (PRISMA) statements [15] (Table S1) focused on vitamin B2, B6 and B12 intake and the polymorphisms of MTHFR (where data were collected from cohort and case-control studies, respectively), and their effects on colorectal cancer risk in adults.

\section{Literature Search}

We carried out a systematic scientific literature search in PubMed, Ovid-Medline, Web of Science (WOS) and ProQuest electronic databases to identify observational studies presenting results on the relationship between B vitamin intake and colorectal cancer risk. Searches were accomplished in all available years until 15th March 2021. We collected publications based on combinations of the following searching terms: B vitamins, vitamin B2, vitamin B6, vitamin B12, colorectal cancer and dietary intake (i.e. PubMed: $B$ vitamins AND colorectal cancer, vitamin B2 AND colorectal cancer, vitamin B6 AND colorectal cancer, vitamin B12 AND colorectal cancer; Ovid-Medline: vitamin B and colorectal cancer and dietary intake; Web of Science/ProQuest: vitamin B2 and colorectal cancer, vitamin B6 and colorectal cancer, vitamin B12 and colorectal cancer). We used advanced search in case of Ovid-Medline, Web of Science, and ProQuest. Electronic search, study selection and review of selected papers were undertaken by two independent authors.

\section{Study Selection and Quality Assessment}

Identified records were screened by titles and abstracts and after removal of duplicated studies, publications were reviewed based on inclusion and exclusion criteria. Inclusion criteria were:

1. Publications had to be written in English.

2. Papers had to be original articles.

3. Patients had to be adults.

4. The exposure of interest was vitamin B2, B6 and B12.

5. The outcome of interest was the diagnosis of colorectal cancer. All studies with only animal or in vitro experiments were excluded. After screening process, the remained 35 studies were assessed by eligibility criteria, which were: 1 . odds ratio (OR), relative risk (RR) or hazard ratio (HR) with 95\% confidence interval (CI) had to be calculated in the article; 2 . the studies had to be cohort or case-control studies (these only were accepted if they discussed the association between B2, B6 and B12 vitamin intake and MTHFR polymorphism in CRC. Articles, which met all the criteria were reviewed again and these publications formed the basis of our quantitative analysis. We applied the Newcastle-Ottawa Scale (NOS) for assessing the quality of included publications in our meta-analysis [16].

\section{Statistical Analysis}

We summarized the observed treatment effect sizes including odd ratios (ORs), confidence intervals (CIs) and weights of 
the studies using random effects model [17-19]. Overall ORs (combined effect size, CES) and the corresponding 95\% CIs and 95\% prediction intervals (PIs) were calculated. The studies were tested using I2 statistic and Cochran's Q test. In order to identify possible sources of heterogeneity, we explored studies with outlier effect sizes using funnel plot and Galbraith plot [20]. We also used the "Trim and fill" method within funnel plot to estimate true effect size and the dispersion of the combined effect size (heterogeneity) [19]. In this process both observed and adjusted combined effects size (CES) were calculated with related CI and PI, respectively [21,22]. We carried out Egger's regression test [23] and Begg \& Mazumdar's rank correlation test to inspect possible publication bias [24]. Publication and other biases of the individual studies were evaluated according to the information found in the original articles. All statistical analysis were implemented by the tools of
Meta-Essentials [25].

\section{Results}

\section{Literature Search}

A total of 1021 articles (199 from PubMed, 178 from OvidMedline, 624 from WoS and 20 from ProQuest) were identified through the electronic search. After screening titles and abstracts and excluding duplicates, 84 items were reviewed according to inclusion and exclusion criteria. 35 articles went through full-text review of which 9 cohort studies focused on the effects of $B$ vitamin intake on CRC risk and further 5 eligible items (case-control studies) discussed the connection between MTHFR polymorphism, CRC risk and B vitamin intake. Finally, 14 eligible studies were included in the quantitative analysis. The selection procedure is presented on the detailed PRISMA flow diagram (Figure 1).

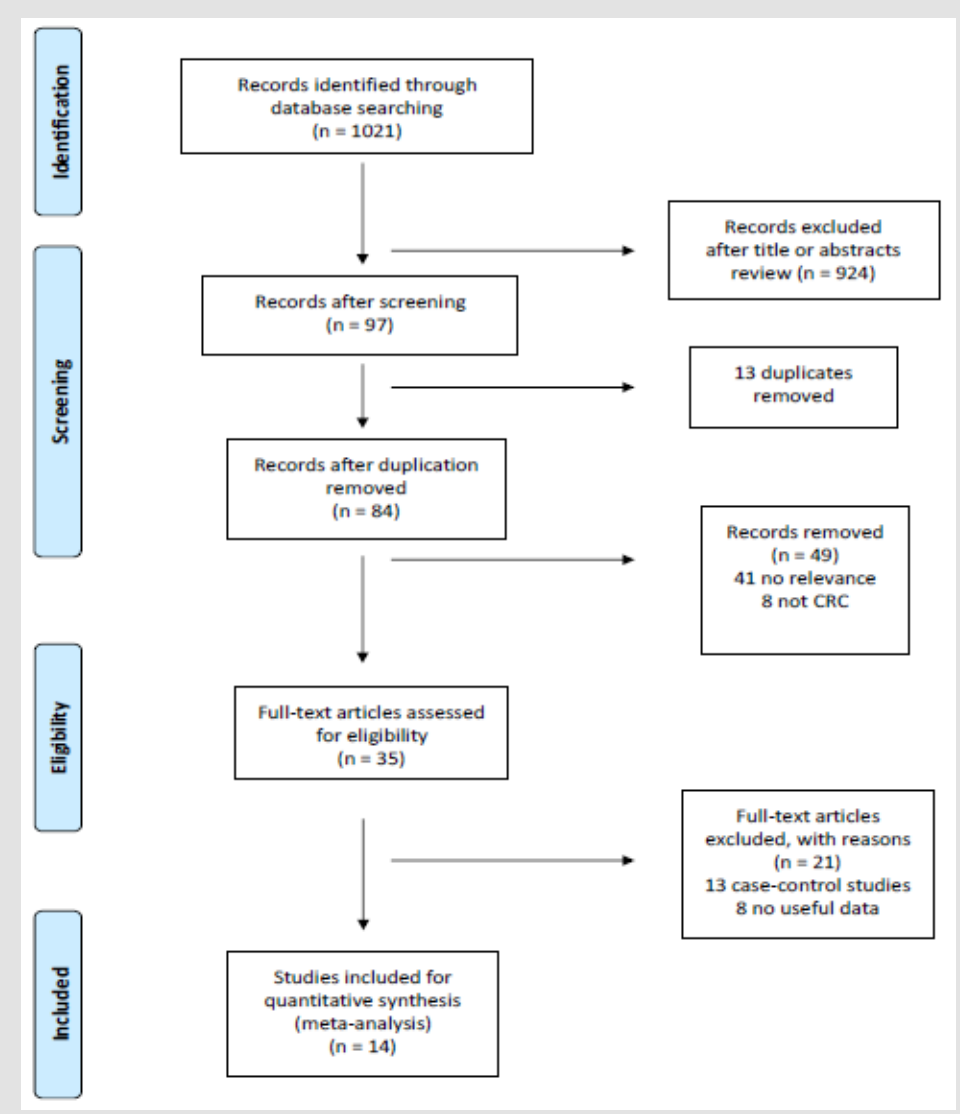

Figure 1: PRISMA flow diagram of study selection for meta-analysis.

\section{Study Characteristics}

In the first analysis consisting of 9 selected articles, we calculated overall ORs for vitamin B2, B6, B12 intake and CRC risk without consideration of MTHFR polymorphism. These studies were cohort studies, 5 from America, 1 from Sweden, China, Japan and Australia. The overall sample size was 777117 and number of cases was 8146 (Table 1). We stratified the analysis according to the type of B vitamin and individual forest plots were generated for vitamin B2, vitamin B6 and vitamin B12 with 5 [26-30], 7 [2729,31-34] and 4 [27-29,34] cohort studies, respectively (Figure $2)$. In the second analysis we evaluated the 5 eligible articles [13,14,35-37] (Figure 3A). Calculated overall OR represented the association between MTHFR C667T homozygous polymorphism 
and B vitamins, influencing the risk of CRC development caused by this gene variant. Regarding study design, these were case-control studies conducted mainly in Europe and the US. The 5 studies had a total of 7790 participants with 2230 cases (Table 2). The daily intake of B vitamins was categorized into low or high groups, using tertiles, quartiles or quintiles. Dose of intake varied between studies (Tables 1 \& 2); therefore, we compared the highest versus lowest intake and related ORs in all cases.

Table 1: List and characteristics of publications, discussing the intake of vitamin B2, B6, B12 and the risk of colorectal cancer, included in the meta-analysis.

\begin{tabular}{|c|c|c|c|c|c|c|c|c|c|}
\hline $\begin{array}{c}\text { Date of } \\
\text { Publication }\end{array}$ & Study(B2) & $\begin{array}{c}\text { OR } \\
(\mathrm{C} 195 \%)\end{array}$ & $\begin{array}{l}\text { Sample } \\
\text { Size }\end{array}$ & Cases & $\begin{array}{c}\text { Daily Dose- } \\
\text { mg/d }\end{array}$ & Adjusted Variables & Study type & Population & $\begin{array}{l}\text { Study } \\
\text { Quality } \\
\text { (NOS) }\end{array}$ \\
\hline 2008 & $\begin{array}{c}\text { Kabat GC et al. } \\
\text { [26] }\end{array}$ & $\begin{array}{c}0.94 \\
(0.73 ; 1.23)\end{array}$ & 49654 & 617 & $\begin{array}{l}<1.56(\mathrm{Q} 1) \\
>1.93(\mathrm{Q} 3)\end{array}$ & $\begin{array}{c}\text { Age (Continuous), Body mass index } \\
\text { (kg/m2-continuous), pack-years of } \\
\text { smoking (none,40tool10.10 too20,20 } \\
\text { too30,30+), years of education (3 } \\
\text { levels), menopausal status (pre-, } \\
\text { peri-, post),oral contraceptive use } \\
\text { (never, ever), hormone replacement } \\
\text { therapy (never, ever), and intake of } \\
\text { calories(continuous).In addition, } \\
\text { all nutrients except alcohol } \\
\text { were adjusted for alcohol intake } \\
\text { (Continuous) }\end{array}$ & Cohort & $\begin{array}{l}\text { Canadian } \\
\text { Women }\end{array}$ & 8 \\
\hline 2009 & $\begin{array}{c}\text { Shrubsole et al. } \\
\text { [27] }\end{array}$ & $\begin{array}{c}1.10 \\
(0.70 ; 1.90)\end{array}$ & 72861 & 394 & $\begin{array}{l}0.62(\mathrm{Q} 1) \\
1.22(\mathrm{Q} 5)\end{array}$ & $\begin{array}{c}\text { Age, educational, smoking status, PA, } \\
\text { baseline household income, drinking } \\
\text { status, HRT menopausal status, } \\
\text { family history of CRC, BMI, NSAID } \\
\text { use, use of a B vitamin supplement, } \\
\text { history of colorectal polyps, diabetes } \\
\text { history, and daily intakes of energy, } \\
\text { vegetables, fruits, red meats, and } \\
\text { calcium }\end{array}$ & Cohort & China & 7 \\
\hline 2013 & $\begin{array}{l}\text { Zschabitz S et } \\
\text { al. [28] }\end{array}$ & $\begin{array}{c}0.81 \\
(0.66 ; 0.99)\end{array}$ & 86820 & 808 & $\begin{array}{l}\leq 1.37(\mathrm{Q} 1) \\
>2.43(\mathrm{Q} 4)\end{array}$ & $\begin{array}{l}\text { BMI, PA, smoking status, aspirin use, } \\
\text { hormone replacement therapy (only } \\
\text { in NHS), multivitamin supplement } \\
\text { use, family history of CRC, and history } \\
\text { of sigmoidoscopy/colonoscopy }\end{array}$ & Cohort & US & 7 \\
\hline 2013 & $\begin{array}{c}\text { Basset JK et al. } \\
\text { [29] }\end{array}$ & $\begin{array}{c}0.89 \\
(0.72 ; 1.11)\end{array}$ & 37112 & 910 & $\begin{array}{l}1.64(\mathrm{Q} 1) \\
3.43(\mathrm{Q} 5)\end{array}$ & $\begin{array}{l}\text { Age, sex, education, smoking status, } \\
\text { education, PA, country of birth, } \\
\text { alcohol consumption, family history } \\
\text { of cancer, intake of cereal fiber }\end{array}$ & Cohort & Australian & 9 \\
\hline 2016 & $\begin{array}{c}\text { Yoon et al. Male } \\
{[30]}\end{array}$ & $\begin{array}{c}0.93 \\
(0.79 ; 1.10)\end{array}$ & M:44007 & 944 & $\begin{array}{c}1.80(\mathrm{Q} 1) \\
15.90(\mathrm{Q} 5)\end{array}$ & $\begin{array}{l}\text { BMI, PA, smoking status, aspirin use, } \\
\text { hormone replacement therapy (only } \\
\text { in NHS), multivitamin supplement } \\
\text { use, family history of CRC, and history } \\
\text { of sigmoidoscopy/colonoscopy }\end{array}$ & Cohort & US & 8 \\
\hline 2016 & $\begin{array}{l}\text { Yoon et al. } \\
\text { Female [30] }\end{array}$ & $\begin{array}{c}0.91 \\
(0.71 ; 1.17)\end{array}$ & $F: 100033$ & 2093 & $\begin{array}{c}1.60(\mathrm{Q} 1) \\
14.30(\mathrm{Q} 5)\end{array}$ & $\begin{array}{l}\text { BMI, PA, smoking status, aspirin use, } \\
\text { hormone replacement therapy (only } \\
\text { in NHS), multivitamin supplement } \\
\text { use, family history of CRC, and history } \\
\text { of sigmoidoscopy/colonoscopy }\end{array}$ & Cohort & US & 8 \\
\hline $\begin{array}{c}\text { Date of } \\
\text { Publication }\end{array}$ & Study(B6) & $\begin{array}{c}\text { OR } \\
\text { (C195\%) }\end{array}$ & $\begin{array}{l}\text { Sample } \\
\text { Size }\end{array}$ & Cases & $\begin{array}{c}\text { Daily Dose- } \\
\text { mg/d }\end{array}$ & Adjusted Variables & Study type & Population & $\begin{array}{l}\text { Study } \\
\text { Quality } \\
\text { (NOS) }\end{array}$ \\
\hline 2005 & $\begin{array}{l}\text { Larsson et al. } \\
\qquad[31]\end{array}$ & $\begin{array}{c}0.96 \\
(0.50 ; 0.86)\end{array}$ & 61433 & 805 & $\begin{array}{l}<1.53(\mathrm{Q} 1) \\
>2.05(\mathrm{Q} 5)\end{array}$ & $\begin{array}{l}\text { Age, education, BMI, intakes of red } \\
\text { meat, saturated fat, calcium, folate, } \\
\text { beta-carotene and cereal fiber }\end{array}$ & Cohort & Sweden & 6 \\
\hline 2005 & $\begin{array}{c}\text { Le Marchande } \\
\text { et al. [32] }\end{array}$ & $\begin{array}{c}0.68 \\
(0.51 ; 0.91)\end{array}$ & $\begin{array}{l}\mathrm{M}: 96810 \\
\mathrm{~F}: 11141\end{array}$ & 383 & $\begin{array}{l}<1.63(\mathrm{Q} 1) \\
>2.64(\mathrm{Q} 5)\end{array}$ & Age, sex, ethnicity & Cohort & US & 6 \\
\hline
\end{tabular}




\begin{tabular}{|c|c|c|c|c|c|c|c|c|c|}
\hline 2007 & $\begin{array}{c}\text { Ishihara J et al. } \\
\text { Male [33] }\end{array}$ & $\begin{array}{c}0.69 \\
(0.48 ; 0.98)\end{array}$ & 38107 & 335 & $\begin{array}{l}1.09(\mathrm{Q} 1) \\
1.91(\mathrm{Q} 4)\end{array}$ & $\begin{array}{c}\text { Age (Continuous), alcohol } \\
\text { consumption (nondrinkers,1-149g/ } \\
\text { wk,150-299g/wk,300 or more g/ } \\
\text { wk), smoking (never, past, current), } \\
\text { BMI (continuous), supplement use } \\
\text { (dichotomous), physical activity } \\
\text { (continuous, MET-h/d), calcium, } \\
\text { vitamin D, meat intake (energy- } \\
\text { adjusted, continuous), and study } \\
\text { area. }\end{array}$ & Cohort & Japan & 7 \\
\hline 2007 & $\begin{array}{c}\text { Ishihara J et al. } \\
\text { Female [33] }\end{array}$ & $\begin{array}{c}1.10 \\
(0.67 ; 1.83)\end{array}$ & 43077 & 191 & $\begin{array}{l}1.02(\mathrm{Q} 1) \\
1.80(\mathrm{Q} 4)\end{array}$ & $\begin{array}{c}\text { Age (Continuous), alcohol } \\
\text { consumption (nondrinkers,1-149g/ } \\
\text { wk,150-299g/wk,300 or more g/ } \\
\text { wk), smoking (never, past, current), } \\
\text { BMI (continuous), supplement use } \\
\text { (dichotomous), physical activity } \\
\text { (continuous, MET-h/d), calcium, } \\
\text { vitamin D, meat intake (energy- } \\
\text { adjusted, continuous), and study } \\
\text { area. }\end{array}$ & Cohort & Japan & 7 \\
\hline 2008 & $\begin{array}{l}\text { Shernhammer } \\
\text { et al. Male [34] }\end{array}$ & $\begin{array}{c}0.82 \\
(0.55 ; 1.22)\end{array}$ & 47371 & 277 & $\begin{array}{l}<1.91(\mathrm{Q} 1) \\
>5.8(\mathrm{Q} 5)\end{array}$ & $\begin{array}{l}\text { Age, BMI, physical activity, smoking, } \\
\text { screening sigmoidoscopy, family } \\
\text { history of colorectal cancer, colon } \\
\text { polyps, aspirin use, multivitamin } \\
\text { use, intakes of alcohol, beef, calcium, } \\
\text { folate, vitamin B12, and methionine }\end{array}$ & Cohort & US & 7 \\
\hline 2008 & $\begin{array}{c}\text { Shernhammer } \\
\text { et al. Female } \\
\text { [34] }\end{array}$ & $\begin{array}{c}0.82 \\
(0.60 ; 1.13)\end{array}$ & 88691 & 389 & $\begin{array}{l}<1.31(\mathrm{Q} 1) \\
>3.5(\mathrm{Q} 5)\end{array}$ & $\begin{array}{l}\text { Age, BMI, physical activity, smoking, } \\
\text { screening sigmoidoscopy, family } \\
\text { history of colorectal cancer, colon } \\
\text { polyps, aspirin use, multivitamin } \\
\text { use, intakes of alcohol, beef, calcium, } \\
\text { folate, vitamin B12, and methionine }\end{array}$ & Cohort & US & 7 \\
\hline 2009 & $\begin{array}{c}\text { Shrubsole et al. } \\
\text { [27] }\end{array}$ & $\begin{array}{c}0.70 \\
(0.40 ; 1.20)\end{array}$ & 72861 & 394 & $\begin{array}{l}1.44(\mathrm{Q} 1) \\
2.33(\mathrm{Q} 4)\end{array}$ & $\begin{array}{c}\text { Age, educational, smoking status, PA, } \\
\text { baseline household income, drinking } \\
\text { status, HRT menopausal status, } \\
\text { family history of CRC, BMI, NSAID } \\
\text { use, use of a B vitamin supplement, } \\
\text { history of colorectal polyps, diabetes } \\
\text { history, and daily intakes of energy, } \\
\text { vegetables, fruits, red meats, and } \\
\text { calcium }\end{array}$ & Cohort & China & 7 \\
\hline 2013 & $\begin{array}{l}\text { Zschabitz S et } \\
\text { al. [28] }\end{array}$ & $\begin{array}{c}0.73 \\
(0.60 ; 0.89)\end{array}$ & 88045 & 808 & $\begin{array}{l}<1.52(\mathrm{Q} 1) \\
>4.00(\mathrm{Q} 4)\end{array}$ & $\begin{array}{l}\text { BMI,PA,smoking status, aspirin } \\
\text { use, hormone replacement therapy } \\
\text { (only in NHS), multivitamin } \\
\text { supplement use, family history of } \\
\text { CRC , and history of sigmoidoscopy/ } \\
\text { colonoscopy }\end{array}$ & Cohort & US & 7 \\
\hline 2013 & $\begin{array}{c}\text { Basset et al. } \\
{[29]}\end{array}$ & $\begin{array}{c}1.01 \\
(0.82 ; 1.23)\end{array}$ & 37112 & 960 & $\begin{array}{l}1.64(\mathrm{Q} 1) \\
3.88(\mathrm{Q} 5)\end{array}$ & $\begin{array}{l}\text { Age, sex, education, smoking status, } \\
\text { education, PA, country of birth, } \\
\text { alcohol consumption, family history } \\
\text { of cancer, intake of cereal fiber }\end{array}$ & Cohort & Australian & 9 \\
\hline $\begin{array}{c}\text { Date of } \\
\text { Publication }\end{array}$ & Study(B12) & $\begin{array}{c}\text { OR } \\
\text { (C195\%) }\end{array}$ & $\begin{array}{l}\text { Sample } \\
\text { Size }\end{array}$ & Cases & $\begin{array}{c}\text { Daily Dose- } \\
\text { mg/d }\end{array}$ & Adjusted Variables & Study type & Population & $\begin{array}{l}\text { Study } \\
\text { Quality } \\
\text { (NOS) }\end{array}$ \\
\hline 2007 & $\begin{array}{c}\text { Ishihara J et al. } \\
\text { Male [33] }\end{array}$ & $\begin{array}{c}1.50 \\
(0.96 ; 2.35)\end{array}$ & 53711 & 106 & $\begin{array}{c}4.2 \mu \mathrm{g}(\mathrm{Q} 1) \\
13.7 \mu \mathrm{g}(\mathrm{Q} 4)\end{array}$ & $\begin{array}{l}\text { Age, education,BMI,intakes of red } \\
\text { meat, saturated fat, calcium, folate, } \\
\text { beta-carotene and cereal fiber }\end{array}$ & Cohort & $\begin{array}{l}\text { Middle aged } \\
\text { Japanese } \\
\text { men and } \\
\text { women }\end{array}$ & 7 \\
\hline 2007 & $\begin{array}{c}\text { Ishihara J et al. } \\
\text { Female [33] }\end{array}$ & $\begin{array}{c}1.70 \\
(0.96 ; 3.01)\end{array}$ & 61136 & 62 & $\begin{array}{c}4.0 \mu \mathrm{g}(\mathrm{Q} 1) \\
12.8 \mu \mathrm{g}(\mathrm{Q} 4)\end{array}$ & $\begin{array}{l}\text { Age, education, BMI, intakes of red } \\
\text { meat, saturated fat, calcium, folate, } \\
\text { beta-carotene and cereal fiber }\end{array}$ & Cohort & $\begin{array}{l}\text { Middle aged } \\
\text { Japanese } \\
\text { men and } \\
\text { women }\end{array}$ & 7 \\
\hline
\end{tabular}




\begin{tabular}{|c|c|c|c|c|c|c|c|c|c|}
\hline 2008 & $\begin{array}{l}\text { Shernhammer } \\
\text { et al. Male [34] }\end{array}$ & $\begin{array}{c}0.66 \\
(0.45 ; 0.97)\end{array}$ & 47371 & 277 & $\begin{array}{l}<0.6(\mathrm{Q} 1) \\
>16.1(\mathrm{Q} 5)\end{array}$ & $\begin{array}{l}\text { Age, BMI, physical activity, smoking, } \\
\text { screening sigmoidoscopy, family } \\
\text { history of colorectal cancer, colon } \\
\text { polyps, aspirin use, multivitamin } \\
\text { use, intakes of alcohol, beef, calcium, } \\
\text { folate, vitamin B12, and methionine }\end{array}$ & Cohort & US & 7 \\
\hline 2008 & $\begin{array}{c}\text { Shernhammer } \\
\text { et al. Female } \\
{[34]}\end{array}$ & $\begin{array}{c}0.95 \\
(0.69 ; 131)\end{array}$ & 88691 & 389 & $\begin{array}{l}<4.0(\mathrm{Q} 1) \\
>11.1(\mathrm{Q} 5)\end{array}$ & $\begin{array}{l}\text { Age, BMI, physical activity, smoking, } \\
\text { screening sigmoidoscopy, family } \\
\text { history of colorectal cancer, colon } \\
\text { polyps, aspirin use, multivitamin } \\
\text { use, intakes of alcohol, beef, calcium, } \\
\text { folate, vitamin B12, and methionine }\end{array}$ & Cohort & US & 7 \\
\hline 2009 & $\begin{array}{c}\text { Shrubsole et al. } \\
\text { [27] }\end{array}$ & $1.3(0.9 ; 1.9)$ & 72861 & 394 & $\begin{array}{l}1.28(\mathrm{Q} 1) \\
4.86(\mathrm{Q} 5)\end{array}$ & $\begin{array}{c}\text { Age, educational, smoking status, PA, } \\
\text { baseline household income, drinking } \\
\text { status, HRT menopausal status, } \\
\text { family history of CRC, BMI, NSAID } \\
\text { use, use of a B vitamin supplement, } \\
\text { history of colorectal polyps, diabetes } \\
\text { history, and daily intakes of energy, } \\
\text { vegetables, fruits, red meats, and } \\
\text { calcium }\end{array}$ & Cohort & China & 7 \\
\hline 2013 & $\begin{array}{c}\text { Basset et al. } \\
\text { [29] }\end{array}$ & $\begin{array}{c}1.05 \\
(0.84 ; 1.31)\end{array}$ & 37112 & 960 & $\begin{array}{l}1.75(\mathrm{Q} 1) \\
5.85(\mathrm{Q} 5)\end{array}$ & $\begin{array}{l}\text { Age, sex, education, smoking status, } \\
\text { education, PA, country of birth, } \\
\text { alcohol consumption, family history } \\
\text { of cancer, intake of cereal fiber }\end{array}$ & Cohort & Australian & 9 \\
\hline 2013 & $\begin{array}{l}\text { Zschabitz S et } \\
\text { al. [28] }\end{array}$ & $\begin{array}{c}0.91 \\
(0.75 ; 1.10)\end{array}$ & 88045 & 808 & $\begin{array}{l}<3.52(\mathrm{Q} 1) \\
>7.27(\mathrm{Q} 4)\end{array}$ & $\begin{array}{l}\text { BMI, PA, smoking status, aspirin } \\
\text { use, hormone replacement therapy } \\
\text { (only in NHS), multivitamin } \\
\text { supplement use, family history of } \\
\text { CRC , and history of sigmoidoscopy/ } \\
\text { colonoscopy }\end{array}$ & Cohort & US & 7 \\
\hline
\end{tabular}

Table 2: List and characteristics of publications discussing the association of vitamin B2 and B6 intake, influenced by MTHFR C667T polymorphism, with the risk of colorectal cancer development included in the meta-analysis.

\begin{tabular}{|c|c|c|c|c|c|c|c|c|c|}
\hline $\begin{array}{c}\text { Date of } \\
\text { publication }\end{array}$ & Study (B2) & OR (CI95\%) & $\begin{array}{l}\text { Sample } \\
\text { size }\end{array}$ & Cases & $\begin{array}{l}\text { Daily dose - } \\
\text { mg/d }\end{array}$ & Adjusted variables & Study type & Population & $\begin{array}{l}\text { Study } \\
\text { quality } \\
\text { (NOS) }\end{array}$ \\
\hline 2005 & $\begin{array}{l}\text { Donk et al. B2 } \\
\text { high TT }\end{array}$ & $\begin{array}{l}0.32(0.16 \\
0.67)\end{array}$ & 1452 & 77 & $\begin{array}{l}\text { low }<1.27 \mathrm{mg} \\
\text { high }>1.92 \mathrm{mg}\end{array}$ & $\begin{array}{l}\text { adjusted for age and dietary folate } \\
\text { and calcium intake }\end{array}$ & case-control & Dutch & 8 \\
\hline 2008 & $\begin{array}{c}\text { Sharp L et al. } \\
\text { [35] B2 high } \\
\text { CT/TT }\end{array}$ & $\begin{array}{l}1.01(0.59 \\
1.74)\end{array}$ & 645 & 134 & $\begin{array}{l}\mathrm{Q} 1<1.87 \mathrm{mg} \\
\mathrm{Q} 4>2.49 \mathrm{mg}\end{array}$ & $\begin{array}{l}\text { adjusted for sex, age, total energy, } \\
\text { physical activity, family history of } \\
\text { colorectal cancer, regular use of any } \\
\text { NSAID, sex x NSAID interaction term; } \\
\text { model for protein also adjusted for } \\
\text { type of dietary supplement; model } \\
\text { for alcohol also adjusted for type of } \\
\text { dietary supplement and protein }\end{array}$ & $\begin{array}{l}\text { case-control } \\
\text { (population- } \\
\text { based: high } \\
\text { CRC, low } \\
\text { folate) }\end{array}$ & Scotland, UK & 7 \\
\hline 2005 & $\begin{array}{l}\text { Donk et al. B2 } \\
\text { high CC }\end{array}$ & $\begin{array}{l}0.57(0.36 \\
0.90)\end{array}$ & 1452 & 336 & $\begin{array}{l}\text { low }<1.27 \mathrm{mg} ; \\
\text { high }>1.92 \mathrm{mg}\end{array}$ & $\begin{array}{l}\text { adjusted for age and dietary folate } \\
\text { and calcium intake }\end{array}$ & case-control & Dutch & 8 \\
\hline 2008 & $\begin{array}{l}\text { Sharp L et al. } \\
\text { [35] B2 high } \\
\text { CC }\end{array}$ & $\begin{array}{l}1.19(0.68 \\
2.09)\end{array}$ & 645 & 117 & $\begin{array}{l}\mathrm{Q} 1<1.87 \mathrm{mg} \\
\mathrm{Q} 4>2.49 \mathrm{mg}\end{array}$ & $\begin{array}{l}\text { adjusted for sex, age, total energy, } \\
\text { physical activity, family history of } \\
\text { colorectal cancer, regular use of any } \\
\text { NSAID, sex NSAID interaction term; } \\
\text { model for protein also adjusted for } \\
\text { type of dietary supplement; model } \\
\text { for alcohol also adjusted for type of } \\
\text { dietary supplement and protein }\end{array}$ & $\begin{array}{l}\text { case-control } \\
\text { (population- } \\
\text { based: high } \\
\text { CRC, low } \\
\text { folate) }\end{array}$ & Scotland, UK & 7 \\
\hline
\end{tabular}




\begin{tabular}{|c|c|c|c|c|c|c|c|c|c|}
\hline $\begin{array}{c}\text { Date of } \\
\text { publication }\end{array}$ & Study (B6) & OR (CI95\%) & $\begin{array}{l}\text { Sample } \\
\text { size }\end{array}$ & Cases & $\begin{array}{l}\text { Daily dose - } \\
\text { mg/d }\end{array}$ & Adjusted variables & Study type & Population & $\begin{array}{l}\text { Study } \\
\text { quality } \\
\text { (NOS) }\end{array}$ \\
\hline 2008 & $\begin{array}{l}\text { Guerreiro CS } \\
\text { et al. [13] B6 } \\
\text { high TT }\end{array}$ & $\begin{array}{c}1.77(0.67 \\
4.68)\end{array}$ & 396 & 26 & $\begin{array}{l}\text { low }<12.5 \mathrm{mg} \\
\text { high }>12.5 \mathrm{mg}\end{array}$ & $\begin{array}{l}\text { adjusted for age, sex, and colorectal } \\
\text { cancer history }\end{array}$ & case control & Portuguese & 6 \\
\hline 1999 & $\begin{array}{l}\text { Slattery ML } \\
\text { et al. [37] B6 } \\
\text { high TT }\end{array}$ & $\begin{array}{l}0.60(0.40 \\
1.00)\end{array}$ & 3288 & 139 & $\begin{array}{l}\text { low }<0.8 \mathrm{mg} \\
\text { high }>1.21 \mathrm{mg}\end{array}$ & $\begin{array}{c}\text { adjusted for sex, age, BMI, long-term } \\
\text { vigorous physical activity, usual } \\
\text { number of cigarettes smoked per } \\
\text { day, total energy intake, and dietary } \\
\text { fiber }\end{array}$ & case control & US & 7 \\
\hline 2008 & $\begin{array}{c}\text { Theodoratou } \\
\text { E et al. [14] B6 } \\
\text { high TT }\end{array}$ & $\begin{array}{l}0.55(0.16 \\
1.85)\end{array}$ & 2009 & 111 & $\begin{array}{l}\min : 1.16 \mathrm{mg} \\
\max : 8.6 \mathrm{mg}\end{array}$ & $\begin{array}{l}\text { Adjusted for energy (residual } \\
\text { method), energy (included as a } \\
\text { covariate) age, sex, deprivation } \\
\text { score, fiber intake (energy adjusted, } \\
\text { quartiles), alcohol intake (energy } \\
\text { adjusted), smoking (nonsmoker, } \\
\text { current smoker, and former } \\
\text { smoker), BMI, NSAID intake, and } \\
\text { family history of cancer (low and } \\
\text { moderate/ high risk) }\end{array}$ & case control & Scotland, UK & 6 \\
\hline 2008 & $\begin{array}{l}\text { Sharp L et al. } \\
\text { [35] B6 high } \\
\text { CT/TT }\end{array}$ & $\begin{array}{l}0.83(0.49 \\
1.39)\end{array}$ & 645 & 134 & $\begin{array}{l}\mathrm{Q} 1<2.29 \mathrm{mg} \\
\mathrm{Q} 4>3.04 \mathrm{mg}\end{array}$ & $\begin{array}{l}\text { adjusted for sex, age, total energy, } \\
\text { physical activity, family history of } \\
\text { colorectal cancer, regular use of any } \\
\text { NSAID, sex NSAID interaction term; } \\
\text { model for protein also adjusted for } \\
\text { type of dietary supplement; model } \\
\text { for alcohol also adjusted for type of } \\
\text { dietary supplement and protein }\end{array}$ & $\begin{array}{l}\text { case-control } \\
\text { (population- } \\
\text { based: high } \\
\text { CRC, low } \\
\text { folate) }\end{array}$ & Scotland, UK & 7 \\
\hline 2008 & $\begin{array}{l}\text { Guerreiro CS } \\
\text { et al. [13] B6 } \\
\text { high CC/CT }\end{array}$ & $\begin{array}{l}0.83(0.55 \\
1.26)\end{array}$ & 396 & 170 & $\begin{array}{l}\text { low }<12.5 \mathrm{mg} \\
\text { high }>12.5 \mathrm{mg}\end{array}$ & $\begin{array}{l}\text { adjusted for age, sex, and colorectal } \\
\text { cancer history }\end{array}$ & case control & Portuguese & 6 \\
\hline 1999 & $\begin{array}{l}\text { Slattery ML } \\
\text { et al. [37] B6 } \\
\text { high CC }\end{array}$ & $\begin{array}{l}0.90(0.70 \\
1.30)\end{array}$ & 3288 & 673 & $\begin{array}{l}\text { low }<0.8 \mathrm{mg} \\
\text { high }>1.21 \mathrm{mg}\end{array}$ & $\begin{array}{c}\text { adjusted for sex, age, BMI, long-term } \\
\text { vigorous physical activity, usual } \\
\text { number of cigarettes smoked per } \\
\text { day, total energy intake, and dietary } \\
\text { fiber }\end{array}$ & case control & US & 7 \\
\hline 2008 & $\begin{array}{c}\text { Theodoratou } \\
\text { E et al. [14] B6 } \\
\text { high CC }\end{array}$ & $\begin{array}{l}0.68(0.40 \\
1.17)\end{array}$ & 2009 & 447 & $\begin{array}{l}\min : 1.16 \mathrm{mg} \\
\max : 8.6 \mathrm{mg}\end{array}$ & $\begin{array}{l}\text { Adjusted for energy (residual } \\
\text { method), energy (included as a } \\
\text { covariate) age, sex, deprivation } \\
\text { score, fiber intake (energy adjusted, } \\
\text { quartiles), alcohol intake (energy } \\
\text { adjusted), smoking (nonsmoker, } \\
\text { current smoker, and former } \\
\text { smoker), BMI, NSAID intake, and } \\
\text { family history of cancer (low and } \\
\text { moderate/ high risk) }\end{array}$ & case control & Scotland, UK & 6 \\
\hline 2008 & $\begin{array}{l}\text { Sharp L et al. } \\
\text { [35] B6 high } \\
\text { CC }\end{array}$ & $\begin{array}{l}0.71(0.41 \\
1.24)\end{array}$ & 645 & 117 & $\begin{array}{l}\mathrm{Q} 1<2.29 \mathrm{mg} \\
\mathrm{Q} 4>3.04 \mathrm{mg}\end{array}$ & $\begin{array}{l}\text { adjusted for sex, age, total energy, } \\
\text { physical activity, family history of } \\
\text { colorectal cancer, regular use of any } \\
\text { NSAID, sex x NSAID interaction term; } \\
\text { model for protein also adjusted for } \\
\text { type of dietary supplement; model } \\
\text { for alcohol also adjusted for type of } \\
\text { dietary supplement and protein }\end{array}$ & $\begin{array}{l}\text { case-control } \\
\text { (population- } \\
\text { based: high } \\
\text { CRC, low } \\
\text { folate) }\end{array}$ & Scotland, UK & 7 \\
\hline
\end{tabular}

Association between Intake of B Vitamins and the Risk of CRC

We applied combined effect size (CES) to demonstrate the association between the highest versus lowest intakes of vitamin $\mathrm{B} 2, \mathrm{~B} 6, \mathrm{~B} 12$ and the risk of CRC.
Vitamin B2: The combined effect size for the risk of CRC for highest versus lowest categories of vitamin B2 intake was 0.90 with CI95\% 0.83 - 0.97, indicating higher intake of vitamin B2 had inverse association with risk of CRC. There was not difference between CI95\% and PI95\% values. Heterogeneity among studies was not observed $\left(I^{2}=0.00 \% ; p=0.910, \mathrm{PI} 95 \%=0.83-0.97\right)$ 
(Figure 2A). According to the "Trim and fill" method there was also no evidence for heterogeneity in case of vitamin B2, and funnel and Galbraith plots did not show any outliers among effect sizes as well. Egger's regression test $(p=0.202)$ and Begg \& Mazumdar's rank correlation test $(p=0.094)$ showed no possible evidence of publication bias.

Vitamin B6: The results of the meta-analysis showed a reduced risk of CRC development by higher dietary intake of vitamin B6
$(\mathrm{CES}=0.80 ; \mathrm{CI} 95 \% 0.68-0.92) . \mathrm{PI} 95 \%$ value $(0.64-0.96)$ was similar to CI95\%. A low statistical heterogeneity was detected ( $\mathrm{I}^{2}$ $=9.17 \% ; \mathrm{p}=0.359 ;$ PI95\% $0.64-0.96$ ) (Figure 2B). According to the "Trim and fill" method there was no evidence for heterogeneity in case of vitamin B6, and funnel and Galbraith plots did not show any outliers among effect sizes as well. Publication bias was not indicated according to Egger's $(\mathrm{p}=0.880)$ and Begg \& Mazumdar's $(\mathrm{p}=0.174)$ tests.

\section{A.}

B2
1 Kabat GC et al female
2 Shrubsole et al
3 Zschabitz $\mathrm{S}$ et al female
4 Basset JK et al
5 Yoon et al male
6 Yoon et al female
7 Overall: $0.90 \mathrm{Cl}(0.83-0.97)$
$\mathrm{PI} \mathrm{(0.83-0.97)}$
I2: $0.00 \%, p: 0.910$

B.

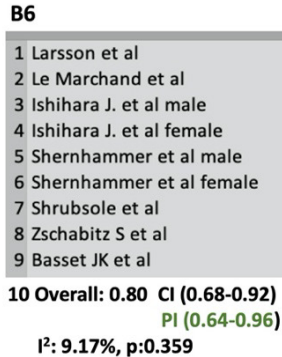

C.

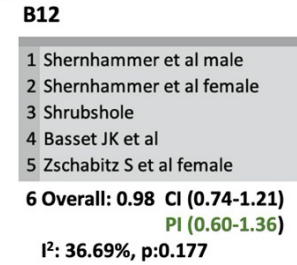

Weighting
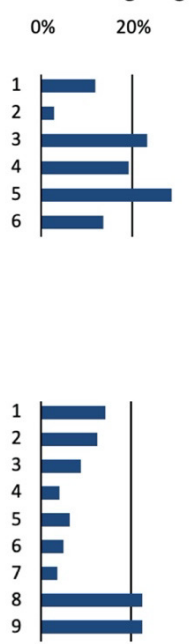

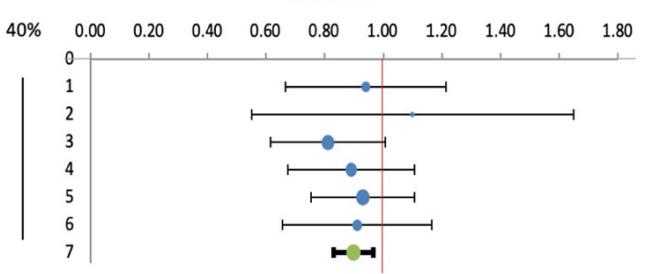

decreased risk of $C R C$

increased risk of CRC

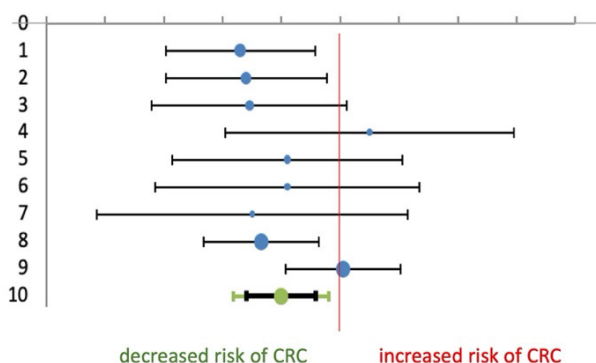

increased risk of CRC
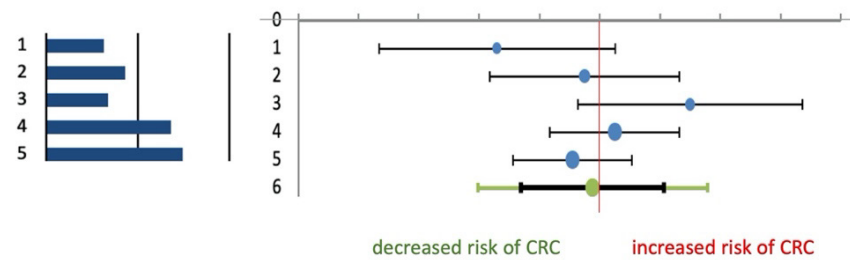

Figure 2: Meta-analysis for the association of vitamin B2 (A), vitamin B6 (B), vitamin B12 (C) intake and colorectal cancer risk. Effect sizes of selected studies, discussing the association of vitamin B intake (highest versus lowest categories) and colorectal cancer, were included. The size of each dot is proportional to the weight of the study.

Vitamin B12: Based on combined effect size calculated from ORs of the 5 selected cohort studies, we observed that higher dietary intake of vitamin B12 could increase the risk of CRC (CES = 1.10; CI95\% 0.80 - 1.39; PI95\% 0.50 - 1.69) in some populations. A significant substantial heterogeneity was presented with $\mathrm{I}^{2}$ $=64.01 \% ; \mathrm{p}=0.011 ; \mathrm{PI} 95 \%=0.50-1.69$. The "Trim and fill" method also showed significant heterogeneity $(p=0.002)$ as well. We visualized effect sizes of vitamin B12 intake to select outliers but neither funnel plot nor Galbraith plot (Figure 3B) suggested outliers, despite the study of Ishihara et al. was more likely to be a possible one. Excluding the results published by Ishihara et al., the meta-analysis on vitamin B12 intake changed significantly.
Based on 4 studies, the recalculated CES fell below 1, changed to 0.98 (CI95\% 0.74 - 1.21; PI95\% 0.60 - 1.36). Heterogeneity became moderate and non-significant $\left(\mathrm{I}^{2}=36.69 \%\right.$; $\left.\mathrm{p}=0.177\right)$ based on the regularly used calculations (Figure 2C), but not on the one proposed by Borenstein. There was no potential publication bias anymore after exclusion ( $\mathrm{p}=0.975$ and $\mathrm{p}=0.500)$.

\section{Association between B Vitamin Intake and MTHFR Polymorphism}

According to random effects model we found that higher dietary intake of vitamin B2 and B6 could decrease the risk of CRC in patients with MTHFR C667T polymorphism. The calculated CES 
was 0.81 with CI95\% $0.64-0.98$ (PI95\% value was the same). Heterogeneity was not detected among the included studies $\left(\mathrm{I}^{2}=0.00 \% ; \mathrm{p}=0.515\right)$ (Figure $\left.3 \mathrm{~A}\right)$. There was also no evidence for heterogeneity by "Trim and fill" method as well. We assessed publication bias in which Egger's regression test and Begg \& Mazumdar's rank correlation test did not show publication bias with levels of significance 0.759 and 0.340 , respectively.

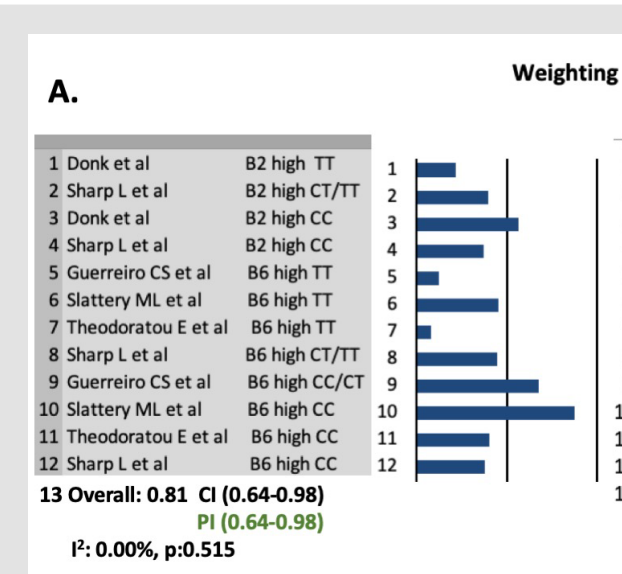

B.

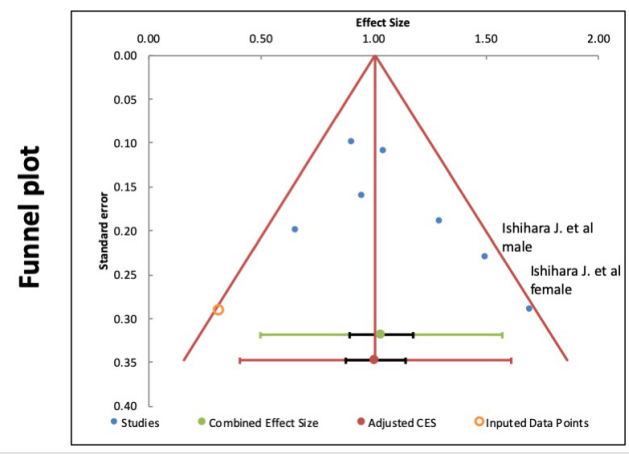

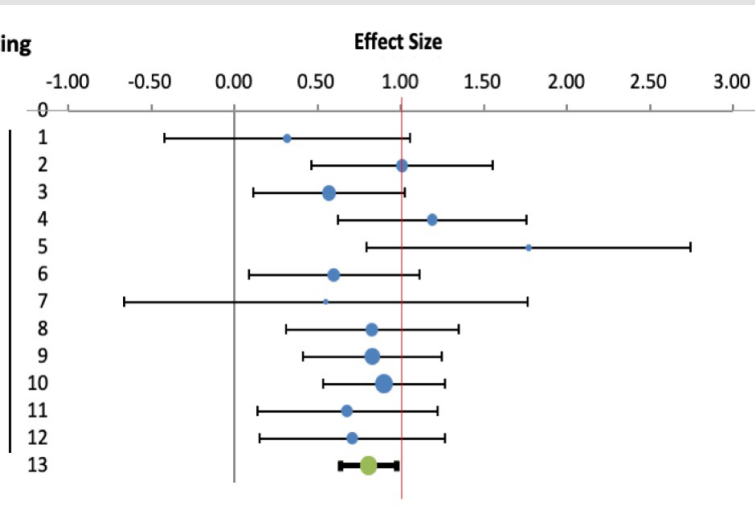

decreased risk of CRC increased risk of CRC

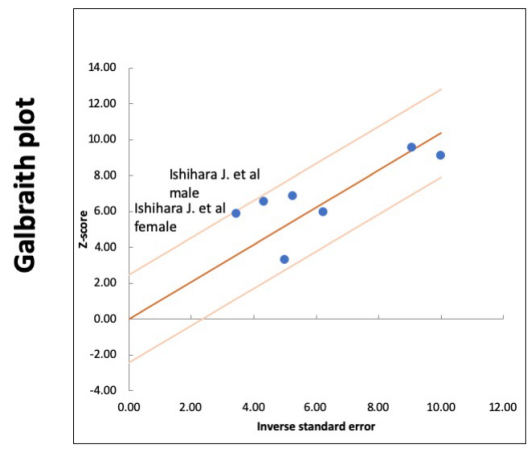

Figure 3: (A) Meta-analysis for the influence of MTHFR C667T polymorphism on the association of B vitamin intake and the risk of CRC. Effect sizes of selected studies, discussing colorectal cancer risk and vitamin B2 and B6 intake, were included. The size of each dot is proportional to the weight of the study. (B) Identification of outliers among studies addressing vitamin B12. Studies outside the skew boundary line of funnel or Galbraith plots are possible outliers.

\section{Discussion}

The importance of nutritional vitamin and mineral intake has increased over the last three decades parallel with the negative environmental factors affecting the human body. Lifestyle factors such as diet, physical activity, stress level and habits, which influenced by social and economic state can increase the risk of cancers. Nutrition of cancer patients requires more attention because their nutritional status is determinative not only for successful cancer treatment but to maintain their physical strength, general well-being or to reduce side effects of their therapies. Therefore, there is an expectation and necessity to measure and evaluate the effects of these vitamins, compounds and products $[6,7,10,38]$. Several studies suggested that dietary methyl-donors and related vitamins can contribute to cancer prevention [8,39-41]. Dietary methyl-donors, such as folate, betaine, choline,methionine and $\mathrm{B}$ vitamins provide methyl groups for the one-carbon metabolism of which vitamin B2, B6 and B12 can influence the availability of methyl groups $[38,7,10]$. B vitamins, additionally, take part in energy-yielding metabolism, oxygen transport and neuronal functions thus they affect the cognitive and psychological processes, including mental and physical fatigue $[7,11]$.

We performed a systematic review and meta-analysis to collect recently available scientific data about the effect of dietary intake of vitamin B2 (riboflavin), B6 (pyridoxine) and B12 (cobalamin) on the risk of CRC development as well as their importance in counteracting MTHFR C677T polymorphism and consequently decrease the risk of CRC development [13,14,35-37]. Although there are well known protocols how to prepare a systematic review or meta-analysis, the interpretation of the results is varied by papers and by selected research area. Most analysis use Cochrane $\mathrm{Q}$ p value and I2 statistics, applying subgroup analysis and calculate heterogeneity as well as publication bias. Heterogeneity regularly 
interpreted as low, moderate, substantial as follows: 30-60\%, 50$90 \%$ and $75-100 \%$, respectively. However, we used additional measurements, the PI95\% as well, to interpret our findings according to Michael Borenstein's recently published book entitled "Common mistakes in Meta-analysis and how to avoid them" [19].

Our meta-analysis suggests a decreased risk of CRC for the highest versus the lowest intake of vitamin B2 and B6. Overall effect was determined as combined effect sizes (CES) with the related CI95\% values. In general, if overall effect size is above 1 , it means the risk increases, when it is placed below 1 that means the risk of CRC decreases. Our results showed that the values of CES are 0.90 for vitamin B2 and 0.80 for B6, thus these vitamins could decrease the risk of CRC. However, there are two additional, regularly used metrics in a meta-analysis, the $\mathrm{I}^{2}$ and the $\mathrm{p}$ value. In a regular basis these are used to evaluate the heterogeneity reflecting on how much the effect sizes varies. However, Borenstein explains that $\mathrm{I}^{2}$ is a ratio and describes us "what proportion of the variance in observed effects reflects variation in true effects, rather than sampling error", and does not say anything about the heterogeneity. In case of heterogeneity, it is more important to answer the question: "how much the true effect size varies across the studies", and the measurement called prediction interval (PI) are able to depict it. In our cases, the PI 95\% values are 0.83 - 0.97 for vitamin B2 and $0.64-0.96$ for vitamin B6. PI95\% does not crossing 1 that means the true effect sizes are below 1 , and as the interval is quite small, it means there is no heterogeneity in these studies.

With regard to the association between vitamin B12 and the risk of CRC, the analysis of the 5 included cohort studies showed that CES is 1.10 with CI95\% $0.80-1.39$ and PI95\% 0.50 - 1.69. The range of PI95\% crossing 1, which suggests that dietary intake of vitamin B12 could increase the risk of CRC in some populations. I2 was $64.01 \%(p=0.011)$, which is considered as a high variance between effect sizes. As a result of the identification of outliers in ORs, we excluded the effect sizes published by Ishihara et al. Even though ORs of this study were inside the skew boundary line of funnel and Galbraith plots, our calculation suggested it is a possible outlier because the I 2 reduced to $36.69 \%(p=0.177)$ after exclusion Although CES changed to 0.98, CI95\% and PI95\% still passed through 1 . This suggested that we still could claim that vitamin B12 could has a negative effect on the risk of CRC in some populations because the range of PI95\% suggested high heterogeneity.

Some publication has already been written that patients in the higher quartile of vitamin B12 intake had more chance to smoking and drinking alcohol, and because of this the utilization of vitamin B12 is decreased in their case [42,43]. As stated by Ishihara et al., there is possibility for positive association between vitamin B12 intake and the risk of CRC, written in their study, which remained after the adjustment of smoking habits and alcohol intake. Therefore, their result represents more likely the effect of smoking and alcohol consumption on the risk of CRC, which is a well-known positive association, rather than the dietary intake of vitamin B12 [33]. All the smoking habits, alcohol consumption and gastrointestinal disorders should be considered if we examine the effect of vitamin B12 intake on the risk of CRC as these factors make it difficult to involve patients properly into any study group based only on their known vitamin B12 intake $[33,44]$. This information led us to exclude vitamin B12 intake from the further analysis. After the exclusion of the study of Ishihara et al. the group of the studies became homogeneous, which is essential criterion for calculating publication bias.

Genetic polymorphisms also can influence the risk of CRC. The most well-known is the single nucleotide polymorphism of MTHFR gene at the position in C677T. This substitution is resulted in decreased enzyme activity in homozygous TT mutation with lower DNA methylation level, thereby increased risk of CRC, however it highly depends on nutritional status [12-14]. Vitamin B2 is the cofactor of MTHFR, which catalyses the formation of 5,10-methyltetrahydrofolate (5,10-THF), and through S-adenosylmethionine (SAM) influences DNA methylation. Depletion of vitamin B2 or folate causes inadequate formation of 5,10-THF and leads to increased homocysteine / S-adenosylhomocysteine (SAH) level and insufficient methylation of DNA, which increases the possibility of development of cancer $[6,45,46]$. Vitamin B6 is a cofactor of cystathionine- $\beta$-synthase which converts homocysteine to cysteine in the liver. Low vitamin B6 level can result in an increased homocysteine and SAM levels, which then similarly can arrest DNA methylation [6,38].

In the second part of our analysis, we investigated the association between MTHFR C667T polymorphism and intake of vitamin B2 and B6. We could confirm that appropriate intake of vitamin $\mathrm{B} 2$ and $\mathrm{B} 6$ could be possibly protective in diminishing or even eliminating the negative effect of the reduced enzyme activity in the folate cycle in case of homozygote TT patients. Additionally, vitamin B2 intake have already been reported as a protective factor for breast and cervical cancer as well, highlighting its potential protective role in cancer prevention [47-49]. There was no evidence for publication bias, indicating that the pooled results may be unbiased. We excluded the group of vitamins B12 from this analysis as well, because the effect of vitamin B12 is influenced by numerous factors as we have already described above. In contrast to the first analysis, in the second, the effect of B vitamins was handled altogether as both vitamin B2 and B6 play role in the onecarbon cycle, which is regulated by MTHFR.

The limitations of our study are similar to other meta-analysis, where several confounding factors (e.g., inadequate controls, misclassification of exposure when using FFQ, dietary intake 
obtained at baseline may have changed over the long follow-up period, high intake of vitamins may have been at lower risk due to other healthy habits and behaviors, adjusted variables differed in the studies) could affect the pooled result. Additionally, nutrients which was not measured in the studies could influence the risk of CRC even after an adjustment process. Details of other possible biases were described in the original papers. We used more searching engines to increase the chance for achieve the highest amount of searching terms related to our analysis as possible. We used additional metrics from Borenstein, which gives additional, valid and meaningful interpretation of the results.

In conclusion, we found that vitamin B2 and B6 may be an effective dietary component to decrease CRC risk, and they can be an important part of a dietary intervention, or a special diet during/ after cancer treatment. We found that an adequate intake of vitamin B2 and B6 - and probably B12 - could compensate the consequence of the reduced enzyme activity of MTHFR in CRC development. Therefore, it may give the opportunity to incorporate a genetic test of the MTHFR polymorphism into the screening process of CRC with recommendations for specific diet for those in need.

\section{Declaration}

\section{Ethical Statement - Not Applicable}

Ethical approval was not sought for this study because of its design (systematic review \& meta- analysis).

\section{Data Availability Statements}

The data that support the findings of this study are openly available in the references number 13-14, 26-37.

\section{Acknowledgement}

We would like to thank to Ferencne Analovics, our librarian for her support and kind guidance how to access online databases through the online library of the university.

\section{Conflict of Interest}

The authors declare no potential conflicts of interest.

\section{Funding Statement}

The study was supported by the NVKP_16-1-2016-0042 grant and by the ÚNKP-21-4-I-SE-19 predoctoral research fellowship by the National Research, Development and Innovation Office of Hungary.

\section{Author Contribution}

EK: literature search, data collection, data analysis, visualization, paper writing, paper review, funding; DM: data collection; RM: paper review; MD: funding, paper review; TI: resources, paper review; ZN: conception, literature search, data collection, data analysis, visualization, paper writing and final approval.

\section{References}

1. Affret A, His M, Severi G, Mancini FR, Arveux P, et al. (2018) Influence of a cancer diagnosis on changes in fruit and vegetable consumption according to cancer site, stage at diagnosis and socioeconomic factors: Results from the large E3N-EPIC study. Int J Cancer 143(7): 1678-1687.

2. Demark-Wahnefried W, Rogers LQ Alfano CM, Thomson CA, Courneya KS, et al. (2015) Practical clinical interventions for diet, physical activity, and weight control in cancer survivors. CA Cancer J Clin 65(3): 167-189.

3. Manser CN, Bauerfeind P (2014) Impact of socioeconomic status on incidence, mortality, and survival of colorectal cancer patients: a systematic review. Gastrointest Endosc 80(1): 42-60 e9.

4. Sung H, Ferlay J, Siegel RL, Laversanne M, Soerjomataram I, et al. (2021) Global Cancer Statistics 2020: GLOBOCAN Estimates of Incidence and Mortality Worldwide for 36 Cancers in 185 Countries. CA Cancer J Clin 71(3): 209-249.

5. McDaniel JT, Nuhu K, Ruiz J, Alorbi G (2017) Social determinants of cancer incidence and mortality around the world: an ecological study. Global Health Promotion 0(0): 1- 9.

6. Mahmoud AM, Ali MM (2019) Methyl Donor Micronutrients that Modify DNA Methylation and Cancer Outcome. Nutrients 11(3).

7. Tardy AL, Pouteau E, Marquez D, Yilmaz C, Scholey A (2020) Vitamins and Minerals for Energy, Fatigue and Cognition: A Narrative Review of the Biochemical and Clinical Evidence. Nutrients 12(1).

8. Kiss E, Forika G, Mohacsi R, Nemeth Z, Krenacs T, et al. (2021) MethylDonors Can Induce Apoptosis and Attenuate Both the Akt and the Erk1/2 Mediated Proliferation Pathways in Breast and Lung Cancer Cell Lines. Int J Mol Sci 22(7).

9. Locasale JW (2013) Serine, glycine and one-carbon units: cancer metabolism in full circle. Nat Rev Cancer13(8): 572-583.

10. Anderson OS, Sant KE, Dolinoy DC (2012) Nutrition and epigenetics: an interplay of dietary methyl donors, one-carbon metabolism and DNA methylation. J Nutr Biochem 23(8): 853-859.

11. (2012) NIDDK. Vitamin B. In: LiverTox: Clinical and Research Information on Drug-Induced Liver Injury [Internet]. 2012-. Updated 2016-May9 ed: Bethesda (MD): National Institute of Diabetes and Digestive and Kidney Disease (NIDDK).

12. Davis CD, Uthus EO (2004) DNA methylation, cancer susceptibility, and nutrient interactions.Exp Biol Med (Maywood) 229(10): 988-995.

13. Guerreiro CS, Carmona B, Goncalves S, Carolino E, Fidalgo P, et al. (2008) Risk of colorectal cancer associated with the C677T polymorphism in 5,10- methylenetetrahydrofolate reductase in Portuguese patients depends on the intake of methyl-donor nutrients. Am J Clin Nutr 88(5): 1413-1418.

14. Theodoratou E, Farrington SM, Tenesa A, McNeill G, Cetnarskyj R, et al. (2008) Dietary vitamin B6 intake and the risk of colorectal cancer. Cancer Epidemiol Biomarkers Prev 17(1): 171-182.

15. Page MJ, McKenzie JE, Bossuyt PM, Boutron I, Hoffmann TC, et al. (2021) The PRISMA 2020 statement: an updated guideline for reporting systematic reviews. BMJ 372: n71.

16. Zeng X, Zhang Y, Kwong JS, Zhang C, Li S, et al. (2015) The methodological quality assessment tools for preclinical and clinical studies, systematic review and meta-analysis, and clinical practice guideline: a systematic review. J Evid Based Med 8(1): 2-10.

17. Borenstein M, Hedges LV, Higgins JP, Rothstein HR (2010) A basic introduction to fixed-effect and random-effects models for metaanalysis. Res Synth Methods 1(2): 97-111.

18. Rothstein MBLVHJPTHHR (2009) Introduction to Meta-Analysis. UK: John Wiley \& Sons, Ltd. 
19. Borenstein M (2019) Common Mistakes in Meta-Analysis and How to Avoid Them: US: Biostat Inc.

20. Galbraith RF (1988) Graphical display of estimates having differing standard errors. Technometrics 30(3): 271-281.

21. Duval S, Tweedie R (2000) A nonparametric "trim and fill" method of accounting for publication bias in meta-analysis. Journal of the American Statistical Association 95(449): 89-98.

22. Duval S, Tweedie R (2000) Trim and fill: A simple funnel-plot-based method of testing and adjusting for publication bias in meta-analysis. Biometrics 56(2): 455-463.

23. Egger M, Davey Smith G, Schneider M, Minder C (1997) Bias in metaanalysis detected by a simple, graphical test. BMJ 315(7109): 629-634.

24. Begg CB, Mazumdar M (1994) Operating characteristics of a rank correlation test for publication bias. Biometrics 50(4): 1088-1101.

25. Suurmond R, van Rhee H, Hak T (2017) Introduction, comparison, and validation of Meta- Essentials: A free and simple tool for meta-analysis. Res Synth Methods 8(4): 537- 553.

26. Kabat GC, Miller AB, Jain M, Rohan TE (2008) Dietary intake of selected $\mathrm{B}$ vitamins in relation to risk of major cancers in women. Br J Cancer 99(5): 816-821.

27. Shrubsole MJ, Yang G, Gao YT, Chow WH, Shu XO, et al. (2009) Dietary $B$ vitamin and methionine intakes and plasma folate are not associated with colorectal cancer risk in Chinese women. Cancer Epidemiol Biomarkers Prev 18(3): 1003-1006.

28. Zschabitz S, Cheng TY, Neuhouser ML, Zheng Y, Ray RM, et al. (2013) $B$ vitamin intakes and incidence of colorectal cancer: results from the Women's Health Initiative Observational Study cohort. Am J Clin Nutr 97(2): 332-343.

29. Bassett JK, Severi G, Hodge AM, Baglietto L, Hopper JL, et al. (2013) Dietary intake of B vitamins and methionine and colorectal cancer risk. Nutr Cancer 65(5): 659-667.

30. Yoon YS, Jung S, Zhang X, Ogino S, Giovannucci EL, et al. (2016) Vitamin B2 intake and colorectal cancer risk; results from the Nurses' Health Study and the Health Professionals Follow-Up Study cohort. Int J Cancer 139(5): 996-1008.

31. Larsson SC, Giovannucci E, Wolk A (2005) Vitamin B6 intake, alcohol consumption, and colorectal cancer: a longitudinal population-based cohort of women. Gastroenterology 128(7): 1830-1837.

32. Le Marchand L, Wilkens LR, Kolonel LN, Henderson BE (2005) The MTHFR C677T polymorphism and colorectal cancer: the multiethnic cohort study. Cancer Epidemiol Biomarkers Prev 14(5): 1198-1203.

33. Ishihara J, Otani T, Inoue M, Iwasaki M, Sasazuki S, et al. (2007) Low intake of vitamin B-6 is associated with increased risk of colorectal cancer in Japanese men. J Nutr 137(7): 1808-1814.

34. Schernhammer ES, Giovannuccci E, Fuchs CS, Ogino S (2008) A prospective study of dietary folate and vitamin B and colon cancer according to microsatellite instability and KRAS mutational status. Cancer Epidemiol Biomarkers Prev 17(10): 2895-2898.

35. Sharp L, Little J, Brockton NT, Cotton SC, Masson LF, et al. (2008) Polymorphisms in the methylenetetrahydrofolate reductase (MTHFR) gene, intakes of folate and related B vitamins and colorectal cancer: a case-control study in a population with relatively low folate intake. $\mathrm{Br} \mathrm{J}$ Nutr 99(2): 379-389.

36. Van den Donk M, Buijsse B, Van den Berg SW, Ocke MC, Harryvan JL, et al. (2005) Dietary intake of folate and riboflavin, MTHFR C677T genotype, and colorectal adenoma risk: a Dutch case-control study. Cancer Epidemiol Biomarkers Prev 14(6): 1562-1566.

37. Slattery ML, Potter JD, Samowitz W, Schaffer D, Leppert M (1999) Methylenetetrahydrofolate reductase, diet, and risk of colon cancer. Cancer Epidemiol Biomarkers Prev 8(6): 513-518.

38. Ducker GS, Rabinowitz JD (2017) One-Carbon Metabolism in Health and Disease. Cell Metab 25(1): 27-42.

39. Chen P, Li C, Li X, Li J, Chu R, et al. (2014) Higher dietary folate intake reduces the breast cancer risk: a systematic review and meta-analysis. Br J Cancer 110(9): 2327-2338.

40. Zeng J, Gu Y, Fu H, Liu C, Zou Y, et al. (2020) Association Between Onecarbon Metabolism-related Vitamins and Risk of Breast Cancer: A Systematic Review and Meta- analysis of Prospective Studies. Clin Breast Cancer 20(4): e469-e80.

41. Yang J, Li H, Deng H, Wang Z (2018) Association of One-Carbon Metabolism-Related Vitamins (Folate, B6, B12), Homocysteine and Methionine With the Risk of Lung Cancer: Systematic Review and MetaAnalysis. Front Oncol 8: 493.

42. Crews H, Alink G, Andersen R, Braesco V, Holst B, et al. (2001) A critical assessment of some biomarker approaches linked with dietary intake. Br J Nutr 86(1): S5- 35.

43. Otani T, Iwasaki M, Yamamoto S, Sobue T, Hanaoka T, et al. (2003) Alcohol consumption, smoking, and subsequent risk of colorectal cancer in middle-aged and elderly Japanese men and women: Japan Public Health Center-based prospective study. Cancer Epidemiol Biomarkers Prev 12(12): 1492-1500.

44. Herrmann W, Obeid R (2008) Causes and early diagnosis of vitamin B12 deficiency. Dtsch Arztebl Int 105(40): 680-685.

45. Bo Y, Zhu Y, Tao Y, Li X, Zhai D, et al. (2020) Association Between Folate and Health Outcomes: An Umbrella Review of Meta-Analyses. Front Public Health 8: 550753.

46. Pieroth R, Paver S, Day S, Lammersfeld C (2018) Folate and Its Impact on Cancer Risk. Curr Nutr Rep 7(3): 70-84.

47. Pufulete M, Al-Ghnaniem R, Leather AJ, Appleby P, Gout S, et al. (2003) Folate status, genomic DNA hypomethylation, and risk of colorectal adenoma and cancer: a case control study. Gastroenterology 124(5): 1240-1248.

48. Aili A, Hasim A, Kelimu A, Guo X, Mamtimin B, et al. (2013) Association of the plasma and tissue riboflavin levels with C20orf54 expression in cervical lesions and its relationship to HPV16 infection. PLoS One 8(11): e79937.

49. Agnoli C, Grioni S, Krogh V, Pala V, Allione A, et al. (2016) Plasma Riboflavin and Vitamin B-6, but Not Homocysteine, Folate, or Vitamin B-12, Are Inversely Associated with Breast Cancer Risk in the European Prospective Investigation into Cancer and Nutrition-Varese Cohort. J Nutr 146(6): 1227-1234. 


\section{ISSN: 2574-1241}

DOI: $10.26717 /$ BJSTR.2022.40.006516

Zsuzsanna Nemeth. Biomed J Sci \& Tech Res

(C) (P) This work is licensed under Creative

Submission Link: https://biomedres.us/submit-manuscript.php

$\begin{array}{ll}\text { BIOMEDICAL } & \text { Assets of Publishing with us } \\ \text { RESEARCHES } & \text { - Global archiving of articles } \\ \text { - Immediate, unrestricted online access } \\ \end{array}$

